\title{
EL PAPEL DE LAS UNIVERSIDADES LOCALES EN LA DIFUSION DE INNOVACIONES SOCIALES: EL CASO DEL DISTRITO MUNICIPAL DE QUITO
}

\section{THE ROLE OF LOCAL UNIVERSITIES IN THE DISSEMINATION OF SOCIAL INNOVATIONS: THE CASE OF THE MUNICIPAL DISTRICT OF QUITO}

\author{
Klaus Gierhake \\ Investigador asociado Zentrum für internationale \\ Entwicklungs- und Umweltforschung \\ Universität Giessen (Alemania) \\ gierhake@gmail.com
}

\author{
Carlos Maria Fernandez Jardon \\ Departamento de Economía Aplicada-ECOBAS \\ Universidad de Vigo, Vigo (España) \\ National Research University Higher School of \\ Economics, Perm (Rusia) \\ cjardon@uvigo.es
}

Fecha de recepción: 08/04/2021 - Fecha de aprobación: 06/05/2021

DOI: https://doi.org/10.36995/j.visiondefuturo.2021.26.01.004.es

\section{RESUMEN}

Los conocimientos locales ayudan a generar innovaciones sociales. Las universidades, que forman parte del sistema de innovación territorial, son importantes en la creación de innovaciones, pero su papel en la difusión de las innovaciones sociales es menos conocido. Las políticas sociales establecidas en Ecuador han dado pie a diferentes innovaciones sociales. En particular, el distrito metropolitano de Quito, a partir del plan de desarrollo metropolitano de 2012 a 2022 ha generado un proceso de innovaciones sociales de gran interés. Las universidades locales suelen participar en el proceso de formación de los agentes que participan en el desarrollo de la innovación social. De modo complementario, las universidades locales pueden facilitar la cooperación entre estos agentes. Ambos son elementos esenciales en la difusión de la innovación. Este trabajo analiza la importancia de la colaboración con las universidades locales para difundir las innovaciones sociales en el Distrito Metropolitano de Quito, haciendo uso de una serie de entrevistas. Los resultados señalan que la falta de colaboración con las universidades y los limitados conocimientos existentes en ellas frenaron el desarrollo práctico del plan y su efectividad.

PALABRAS CLAVE: Territorio; Innovación social; Distrito metropolitano de Quito; Colaboración con universidades.

\section{ABSTRACT}

Local knowledge helps generate social innovations. Universities, which are part of the territorial innovation system, are important in creating innovations, but their role in diffusing social innovations is less well known. The social policies established in Ecuador have given

\footnotetext{
"Visión de Futuro" Año 19, Volumen No 26 N 1, Enero - Junio 2022 - Pág 123 - 148

URL de la Revista: http://visiondefuturo.fce.unam.edu.ar/index.php/visiondefuturo/index

URL del Documento: https://visiondefuturo.fce.unam.edu.ar/index.php/visiondefuturo/issue/view/22

ISSN 1668 - 8708 - Versión en Línea

E-mail: revistacientifica@fce.unam.edu.ar
} 
rise to different social innovations. In particular, the metropolitan district of Quito, based on the metropolitan development plan from 2012 to 2022, has generated a process of social innovations of great interest. Local universities usually participate in the training process of the agents involved in the development of social innovation. In a complementary way, local universities can facilitate cooperation between these agents. Both are essential elements in the diffusion of innovation. This paper analyzes the importance of collaboration with universities to disseminate social innovations in the Metropolitan District of Quito, using a series of interviews. The results indicate that the lack of collaboration with the universities and the limited knowledge that exists in them hampered the practical development of the plan and its effectiveness.

KEY WORDS: Territory; Social innovation; Metropolitan District of Quito; Universities collaboration.

\section{INTRODUCCIÓN}

Las transformaciones políticas ocurridas en los últimos años en diferentes países latinoamericanos (Battaglino, 2015) marcaron un cambio en la orientación de los objetivos políticos. En Ecuador, en particular, se estableció una nueva constitución más orientada al desarrollo sostenible (Manosalvas, 2014), que forma parte del plan de acción mundial que las Naciones Unidas aprobaron en la conferencia de Rio de 1992 para las políticas económicas de los países (UN, 1992). El desarrollo sostenible en los territorios suele estar asociado a un proceso innovativo continuo (Ketelhöhn y Ogliastri, 2013), fruto de la existencia de entornos creativos (Butzin, 2000), que posibilitan la generación de riqueza y empleo. Esa es la esencia de las regiones que aprenden.

Los territorios que aprenden están mejor preparados para afrontar los desafíos del futuro, como ocurre con la búsqueda de estrategias de adaptación al cambio climático y de diferentes formas de aprovechamiento sostenible del territorio (Ogden y Innes, 2009). E desarrollo sostenible exige una preocupación por mejorar económica, social y ambientalmente la región combinando los tres aspectos en sus estrategias de desarrollo (Placet et al., 2005). Esas estrategias están condicionadas por limitaciones de recursos y por diferentes riesgos ambientales que afectan especialmente a los países en vías de desarrollo (Williamson et al., 2012). Consecuentemente, en esos países, existe un especial interés por buscar innovaciones sociales que mitiguen los efectos de esos potenciales cambios y de sus limitados recursos. Las innovaciones sociales suelen estar asociadas al territorio, puesto que en él se integran los aspectos económicos, sociales y medioambientales ( $\mathrm{Li}$ y Toppinen, 2011). Existen

\footnotetext{
"Visión de Futuro" Año 19, Volumen No 26 N 1, Enero - Junio 2022 - Pág 123 - 148 URL de la Revista: http://visiondefuturo.fce.unam.edu.ar/index.php/visiondefuturo/index

URL del Documento: https://visiondefuturo.fce.unam.edu.ar/index.php/visiondefuturo/issue/view/22 
diferentes estudios de nivel territorial, fundamentalmente orientados al ámbito regional o nacional (Bernardino y Santos, 2017; Conejero Paz y Redondo Lebrero, 2016; García-Flores y Martos, 2019; Jardon y Gierhake, 2017). Son menos comunes los trabajos orientados al ámbito municipal (Bernardino y Santos, 2017; Wolfe, 2009). Sin embargo, las acciones sociales desarrolladas a un nivel territorial cercano a las personas, como es el caso de los municipios, suelen tener un efecto más permanente y eficaz, puesto que los agentes participantes se sienten más comprometidos (FEMP, 2011). La innovación social en territorios pequeños suele fundamentarse en el sistema local de innovación, puesto que muchas de las externalidades generadas por el sistema afectan positivamente a los agentes económicos y sociales. La interacción entre las innovaciones empresariales y los sistemas locales de innovación ha sido analizado (Dameri y Ricciardi, 2015), pero la relación de ese sistema con las innovaciones sociales necesita una mayor formalización (Bernardino y Santos, 2017; García-Flores y Martos, 2019; Oeij et al., 2019).

Dentro de estos sistemas, trabajos previos han analizado la importancia de la colaboración con universidades y centros de investigación para generar innovaciones (Mingxing et al., 2020) y, más en particular, como las universidades ayudan a crear innovaciones sociales (Conejero Paz y Redondo Lebrero, 2016; García-Flores y Martos, 2019). Sin embargo, la aportación de las universidades locales a la difusión de las innovaciones en el territorio necesita un análisis más profundo.

El concepto de innovación social ha sido analizado en diferentes trabajos (Conejero Paz y Redondo Lebrero, 2016; García-Flores y Martos, 2019; Oeij et al., 2019), si bien las definiciones son muy diversas y existen diferentes enfoques y caracterizaciones (GarcíaFlores y Martos, 2019), que dificultan su estudio. En general, la mayoría de los trabajos coinciden en dos aspectos característicos de la innovación social: su novedad, es decir, una nueva idea que cambia los procesos, los productos, la organización, etc...., común con todas las innovaciones (García-Flores y Martos, 2019); y su orientación social (Conejero Paz y Redondo Lebrero, 2016; Oeij et al., 2019), que es lo que la diferencia esencialmente de otros tipos de innovación. Este segundo aspecto es el más difícil de analizar, puesto que no existe un consenso sobre lo que es un bien social, salvo en algunos casos particulares. Eso dificulta donde aparece una innovación social y donde no.

Una de las características que se suele pedir a la innovación es que sea sustentable, es decir, que cumpla satisfactoriamente sus objetivos desde el punto de vista económico, social y medioambiental (Placet et al., 2005). Muchas de las innovaciones propuestas en diferentes trabajos no asumen la sostenibilidad económica y, en muchos casos, no analizan los aspectos

\footnotetext{
"Visión de Futuro" Año 19, Volumen No 26 N 1, Enero - Junio 2022 - Pág 123 - 148 URL de la Revista: http://visiondefuturo.fce.unam.edu.ar/index.php/visiondefuturo/index

URL del Documento: https://visiondefuturo.fce.unam.edu.ar/index.php/visiondefuturo/issue/view/22

ISSN 1668 - 8708 - Versión en Línea

E-mail: revistacientifica@fce.unam.edu.ar
} 
medioambientales (Wethinq, 2016), por lo que no deberían considerarse innovaciones sociales propiamente dichas. Consecuentemente, los estudios sobre aspectos particulares de la innovación social estén muy condicionados por la definición de innovación social (Conejero Paz y Redondo Lebrero, 2016; Oeij et al., 2019; Wolfe, 2009). En particular, eso ocurre con los estudios sobre los factores que favorecen o limitan la innovación social.

Dentro del bloque más amplio de factores que limitan la innovación social, se encuentran dos aspectos clave: la formación de los agentes participantes y la cooperación existente entre los agentes que participan en el desarrollo de la innovación (García-Flores y Martos, 2019). Estos dos aspectos engloban muchos de los tópicos señalados en estudios previos (Bernardino y Santos, 2017; García-Flores y Martos, 2019; C.M. Jardon y Gierhake, 2017; Wolfe, 2009). En la interacción de esos dos aspectos se encuentra la colaboración con las universidades locales.

El conocimiento local está formado por el conjunto de conocimientos, tradiciones y comportamientos que han ayudado a los habitantes del territorio a adaptarse a los cambios ambientales ocurridos durante su historia (Jardon y Gierhake, 2017). Es, por tanto, un conjunto de activos intangibles asociados al territorio. Esas adaptaciones les han ayudado a mantener un equilibrio en su desarrollo y han permitido que el territorio tenga una cierta sostenibilidad.

El conocimiento local forma parte del capital intelectual regional (Jardon y Gierhake, 2017). El capital intelectual ha sido analizado en países y regiones (Seleim y Bontis, 2013), aunque apenas hay estudios a nivel municipal, posiblemente porque la información disponible no recoge gran parte de los aspectos asociados a ese concepto. Trabajos previos han estudiado el conocimiento local como factor de innovación social (Jardon y Gierhake, 2017). Sin embargo, el mecanismo mediante el cual, el conocimiento local, actúa difundiendo la innovación social no ha sido suficientemente analizado. Una parte del conocimiento local, especialmente, el conocimiento explícito (Brökel, 2016), se encuentra, o se debería encontrar, en las universidades del territorio. Dado que el conocimiento local ayuda a difundir la innovación, se espera que eso ocurra en los territorios donde se producen innovaciones sociales. Sin embargo, las particularidades de la innovación social, por ejemplo, su carácter de bien colectivo (Eizaguirre Anglada, 2017) y los aspectos culturales asociados a su éxito (Mendes et al., 2012) dificultan la difusión de la innovación, lo que puede hacerla fracasar.

La tesis que presentamos en este trabajo es que la existencia de conocimiento local en un territorio fomenta la innovación social, pero si no existe colaboración fluida con las universidades locales, el impacto de la innovación social en el territorio se ve muy limitado. Para comprobar esta tesis, seguimos un proceso de desarrollo de la innovación social, similar

\footnotetext{
“Visión de Futuro" Año 19, Volumen No 26 N 1, Enero - Junio 2022 - Pág 123 - 148

URL de la Revista: http://visiondefuturo.fce.unam.edu.ar/index.php/visiondefuturo/index

URL del Documento: https://visiondefuturo.fce.unam.edu.ar/index.php/visiondefuturo/issue/view/22

ISSN 1668 - 8708 - Versión en Línea

E-mail: revistacientifica@fce.unam.edu.ar
} 
al esquema de las innovaciones empresariales (Oeij et al., 2019) adaptado a la situación de los pequeños territorios. Después, se señala como afecta el conocimiento local a la difusión de las innovaciones sociales, señalando la importancia de la colaboración con universidades locales para llevar a éxito es proceso. La comprobación empírica se lleva a cabo estudiando el caso del Municipio del Distrito Metropolitano de Quito (MDMQ) puesto que las acciones desarrolladas mediante el Plan de Desarrollo Metropolitano Quito 2012-2022 (MDMQ, 2012c) y sus instrumentos (MDMQ, 2012b, 2012c, 2012a) muestran un ejemplo de innovación social a nivel municipal, en la que intervienen elementos del sistema local de innovación, de tal forma que facilitan la comprensión del alcance de las innovaciones sociales y sus consecuencias a nivel político, económico y social.

El trabajo combina un doble enfoque. Por una parte, hace uso de un enfoque sectorial que analiza el proceso de innovación desde un punto de vista más económico, considerando los diferentes elementos que componen los sistemas regionales de innovación (Lau y Lo, 2015). Por otra parte, hace uso de un enfoque territorial holístico, desde un punto de vista más geográfico, considerando aspectos de la geografía de la innovación (Jardon y Gierhake, 2020).

El esquema del trabajo continúa de la siguiente forma: En primer lugar, se estudia el marco teórico de las barreras para la difusión de la innovación social y su conexión con el conocimiento local, analizando dichos conceptos y su relación en el territorio. A continuación, se expone la metodología, Después, se analiza el caso del DMQ desde las teorías expuestas previamente, con idea de mostrar los efectos que el conocimiento local tiene sobre las barreras a la innovación social. Finalmente, se exponen las conclusiones.

\section{DESARROLLO}

\section{Las innovaciones sociales}

El concepto de innovación social presenta múltiples acepciones y enfoques que dificultan su formalización (Conejero Paz y Redondo Lebrero, 2016; García-Flores y Martos, 2019; Oeij et al., 2019; Wolfe, 2009). En general, en todas las definiciones se perciben dos elementos clave para definir este concepto asociados a la idea de novedad y a la idea de mejora social. La idea de novedad está implícita en el propio concepto de innovación, es decir, "la concepción e implantación de cambios significativos ... con el propósito de mejorar los resultados" (OECD, 2005, p.44). La idea de mejora social es más compleja y difícil de analizar, puesto que puede estar condicionada por ideologías y llevar asociado implicaciones políticas

\footnotetext{
"Visión de Futuro" Año 19, Volumen No 26 N 1, Enero - Junio 2022 - Pág 123 - 148 URL de la Revista: http://visiondefuturo.fce.unam.edu.ar/index.php/visiondefuturo/index

URL del Documento: https://visiondefuturo.fce.unam.edu.ar/index.php/visiondefuturo/issue/view/22

ISSN 1668 - 8708 - Versión en Línea

E-mail: revistacientifica@fce.unam.edu.ar
} 
y sociales que no son compartidas por toda la sociedad y, en muchos casos, ni siquiera por una mayoría. Esto dificulta el uso de este concepto en la práctica. Los autores suelen paliar esa dificultad haciendo uso de conceptos genéricos. Por ejemplo, Rodríguez Herrera y Alvarado Ugarte (2008) sugieren que las innovaciones sociales deben incluir algún tipo de repercusión social, puesto que debe beneficiar a un grupo significativo de personas, contribuyendo a la reducción de la pobreza o a una mejor calidad de vida de los grupos en riesgo social y, paralelamente, debe ser medioambiental. Estos autores también incluyen su carácter de novedad, cuando sugieren que complementariamente, debe tener la capacidad de transformar algún aspecto de la sociedad. De acuerdo a la concepción que proponemos, se exige que produzca también una mejora económica, otro de los aspectos del desarrollo sustentable (Placet et al., 2005). La innovación social debe ser capaz de difundirse a otras situaciones y lugares, porque debería poder ser reproducida en otro lugar o situación y/o a mayor escala. Las innovaciones sociales tienden por su esencia a su difusión y a su expansión (Rodríguez Herrera y Alvarado Ugarte, 2008). Las innovaciones sociales no pretenden la generación de ventajas sobre competidores y no tienen que ser protegidas por patentes (Morales Gutiérrez, 2009), por consiguiente, pueden ser consideradas innovaciones abiertas. Esto hace que las innovaciones sociales cuenten normalmente con un cierto elemento de intangibilidad y, a la vez, están íntimamente conectadas con el desarrollo sustentable.

La innovación social tiene repercusiones territoriales, dado que tanto su nacimiento como su desarrollo se apoya en los recursos territoriales (García-Flores y Martos, 2019). Dentro de un territorio, todas las innovaciones muestran un proceso dinámico de difusión (Jardon y Gierhake, 2018). En dicho proceso, se suelen distinguir los actores principales, los canales de comunicación, las barreras y el tiempo necesario para que una innovación se mueva en el espacio territorial. Esos aspectos van a ser analizados en MDMQ, de forma que podamos extraer experiencias que nos permitan elaborar una conceptualización teórica que facilite futuras innovaciones sociales.

García-Flores y Martos (2019), partiendo de un estudio empírico, clasifican los factores de irrupción de la innovación en cinco aspectos: factores culturales y sociales de la población, apoyo político e institucional, conocimientos y mecanismos facilitadores, componentes espaciales y entidades y mecanismos que determinan la estructura productiva empresarial y social. Oganisjana et al. (2015) analizan los factores de innovación social sistematizándolos como internos y externos en relación con un individuo o un grupo de individuos. En particular, se centran en las barreras. Las principales barreras internas son las que están asociadas a las personas, como puede ser la mentalidad (Brown y Wyatt, 2010), la resistencia al cambio o competencias poco desarrolladas (Koch y Hauknes, 2005), entre otros elementos. Las

\footnotetext{
“Visión de Futuro" Año 19, Volumen No 26 N 1, Enero - Junio 2022 - Pág 123 - 148 URL de la Revista: http://visiondefuturo.fce.unam.edu.ar/index.php/visiondefuturo/index

URL del Documento: https://visiondefuturo.fce.unam.edu.ar/index.php/visiondefuturo/issue/view/22

ISSN 1668 - 8708 - Versión en Línea

E-mail: revistacientifica@fce.unam.edu.ar
} 
barreras externas están condicionadas a desafíos más complejos tales como un exceso de reglas burocráticas, presiones de entrega del marco administrativo y legal (Clark et al., 2008), ausencia de capacidad para el aprendizaje organizativo en todos los niveles (Mendes et al., 2012), y financiación insuficiente (Koch y Hauknes, 2005; Mendes et al., 2012).

Oeij et al. (2019) enfocan la innovación social como proceso señalando los elementos clave en cada fase. En la fase inicial, lo principal es el compromiso de los grupos de interés; en la fase de planificación, el apoyo financiero e institucional; en la fase de desarrollo, están los acuerdos entre los diferentes participantes y la capacidad para superar los posibles reveses que se presenten. En esta fase, es fundamental el consenso, la capacidad de liderazgo, las relaciones externas y la existencia de infraestructuras que favorezcan el proceso. En la fase de implementación y difusión es clave el valor social de la innovación, la adaptación a lo local y las posibilidades de extensión a otros territorios. La colaboración con las universidades locales aparece como factor de la innovación social como parte del sistema de innovación local (García-Flores y Martos, 2019; Mendes et al., 2012; Oeij et al., 2019), pero no aparecen como un factor clave en la difusión de la innovación social.

\section{Actores del proceso de innovaciones sociales}

Oeij et al. (2019) proponen un modelo de generación de innovaciones sociales similar al de las innovaciones empresariales, presentando tres periodos. Cada uno de ellos incluye diferentes fases y actores participantes. El primer periodo es el de iniciación que se refiere a la creación de la innovación; el segundo es el periodo de desarrollo, que corresponde al proceso de elaboración y estructuración de la innovación; y el ultimo es el proceso de implementación y terminación, en el que se incluye la difusión tras la adopción de la innovación. Esta es la fase que presenta mayor interés en este trabajo.

La difusión de innovaciones sociales sigue un proceso similar al de las innovaciones empresariales (Oeij et al., 2019), aunque presenta diferencias, especialmente, en la importancia que los actores participantes en esa innovación tienen en su desarrollo ( Gierhake y Jardon, 2017). Los modelos tradicionales de innovación empresarial señalan que los actores de la innovación son las empresas, como actor principal, el sector público y los centros de investigación (Abbas et al., 2019). Estos actores también pueden intervenir en la innovación social, pero esta incluye otros actores de mayor importancia. Gierhake (2015) propone los gobiernos local y nacional, las universidades, la sociedad civil y los actores de cooperación internacional como actores fundamentales en el proceso de creación y difusión de la innovación. En la innovación social, el actor principal puede ser una persona, una institución

\footnotetext{
"Visión de Futuro" Año 19, Volumen No 26 N 1, Enero - Junio 2022 - Pág 123 - 148

URL de la Revista: http://visiondefuturo.fce.unam.edu.ar/index.php/visiondefuturo/index

URL del Documento: https://visiondefuturo.fce.unam.edu.ar/index.php/visiondefuturo/issue/view/22

ISSN 1668 - 8708 - Versión en Línea

E-mail: revistacientifica@fce.unam.edu.ar
} 
o un grupo de personas o instituciones, puesto que suele incluir la necesidad de cooperar entre los actores. Por tanto, cada uno de los actores citados previamente (gobiernos, universidades, etc..) puede formar parte del actor principal de la innovación o puede ser soporte para su desarrollo y difusión.

El proceso de difusión de la innovación suele ser fruto de la interacción entre estos actores siendo especialmente importante su interacción con el actor principal de la innovación. Ese proceso presenta dos fases esenciales en la innovación social. Una primera fase corresponde a la difusión en el territorio. Las innovaciones sociales habitualmente están dirigidas a resolver problemas locales asociados al territorio por lo que su conexión con el territorio es esencial. Los beneficiarios directos suelen ser agentes locales. Consecuentemente, el primer paso en la difusión consiste en que estos beneficiarios acepten la innovación y la asuman como propia para que esta tenga repercusiones sobre ellos. Una segunda fase, más común con otras innovaciones, es la difusión internacional y su extensión a otros territorios. Las ideas incluidas en la innovación social suelen presentar aspectos comunes que sirven para solucionar problemas de otros lugares en los que esa innovación puede aplicarse, bien directamente o bien adaptándola. En ese sentido, la difusión de la innovación necesita ser conocida en el territorio y asumida por los posibles beneficiarios de la innovación y luego, o paralelamente, darla a conocer a otros territorios para su posible difusión. De ahí la importancia de los aspectos geográficos asociados a la innovación social (Gierhake y Jardón, 2016).

En ese proceso de difusión, las principales barreras a la innovación social surgen precisamente por las dificultades existentes en la interacción entre los diferentes actores del proceso. El modelo de difusión presentado muestra una serie de categorías que incluyen la interacción de dos o más actores del proceso de difusión, indicando la actuación de dichos actores en esa interacción y las barreras que pueden presentarse como fruto de esa interacción. Las universidades locales podrían jugar un papel clave en la superación de esas dificultades.

\section{El papel de las universidades locales en la innovación social}

El conocimiento local existente en un territorio presenta dos aspectos complementarios que, en muchos casos, son difíciles de separar. Por una parte, están los conocimientos específicos del territorio, consecuencia de las tradiciones locales, normalmente un conocimiento tácito, que es difícil de formalizar. Por otra parte, existe un conocimiento científico, fruto del saber universal que se trasmite a través de los centros educativos esencialmente y que refuerza los conocimientos locales al interactuar con ellos (Jardon y

\footnotetext{
"Visión de Futuro" Año 19, Volumen No 26 N 1, Enero - Junio 2022 - Pág 123 - 148

URL de la Revista: http://visiondefuturo.fce.unam.edu.ar/index.php/visiondefuturo/index

URL del Documento: https://visiondefuturo.fce.unam.edu.ar/index.php/visiondefuturo/issue/view/22

ISSN 1668 - 8708 - Versión en Línea

E-mail: revistacientifica@fce.unam.edu.ar
} 
Gierhake, 2017). Las universidades locales combinan ambos tipos de conocimientos, si bien dentro del conocimiento local más específico, solo aquello que pueden explicitar es transmitido. La innovación social se apoya, en gran parte, en el conocimiento local por lo que el papel de las universidades es evidente a la hora de participar en la creación de las innovaciones sociales.

El papel de las universidades locales no se suele limitar a la creación, sino que también presenta una función importante en la difusión de la innovación. Por una parte, debe ser capaz de dar la formación necesaria en el territorio para que los agentes locales asuman la innovación y puedan completarla y aplicarla al territorio. Además, pueden ser canales para difundir la innovación entre los agentes locales, a través de los procesos de aprendizaje territorial. Por otra parte, tiene una tarea en la difusión de la innovación a otras universidades, a través de publicaciones, conferencias, seminarios, etc.... donde dan a conocer los aspectos teóricos de la innovación y sus posibilidades de extensión a otros territorios (García-Flores y Martos, 2019; Sáez, 2017). Esto sugiere que, aunque la universidad local no sea el actor principal de la innovación social, puede participar como uno de sus agentes más importantes en su generación, pero, en cualquier caso, suele tener una tarea esencial en su difusión. Las limitaciones existentes en la universidad local van a tener un efecto directo sobre la innovación social generando barreras que, a veces, pueden impedir el proceso de difusión.

El modelo propuesto señala la posibilidad de interacción entre los diferentes componentes del sistema de innovación social. El conocimiento local especifico asociado a la cultura del territorio facilita la interacción entre los actores locales, pero puede dificultar la interacción con actores no locales, nacionales o internacionales. El conocimiento local genérico facilita la interacción con todos los actores, resaltando la importancia del papel de las universidades en el proceso.

En consecuencia, proponemos dos hipótesis que se van a analizar en el DMQ, ambas muy relacionados entre sí:

H1: Las universidades locales juegan un papel preponderante en la difusión de las innovaciones sociales en el territorio.

H2: La falta de colaboración de las universidades locales en el proceso de difusión de una innovación social limita claramente su difusión.

\section{Metodología}

Para analizar una situación geográfica es necesario combinar la información teórica con el análisis territorial. El marco teórico se ha elaborado tras una exhaustiva revisión bibliográfica

\footnotetext{
"Visión de Futuro" Año 19, Volumen No 26 N 1, Enero - Junio 2022 - Pág 123 - 148

URL de la Revista: http://visiondefuturo.fce.unam.edu.ar/index.php/visiondefuturo/index

URL del Documento: https://visiondefuturo.fce.unam.edu.ar/index.php/visiondefuturo/issue/view/22

ISSN 1668 - 8708 - Versión en Línea

E-mail: revistacientifica@fce.unam.edu.ar
} 
y la elaboración de los conceptos que surgen a raíz de los elementos de conocimiento local e innovación social definidos.

El objetivo de este trabajo era describir y explicar el papel de las universidades locales en la difusión de una innovación social desde un enfoque del conocimiento local, por lo que parece más adecuada una metodología cualitativa (Denzin y Lincoln, 2000). En consecuencia, se han utilizado diferentes fuentes de información empírica. Por una parte, se ha revisado la documentación de la municipalidad, tanto sobre el diseño del plan estratégico, como el posterior desarrollo de conocimiento local en cada una de las subunidades organizativas. Por otra parte, se han realizado 40 entrevistas semiestructuradas con los principales actores sociales del proceso y observadores ajenos, expertos que permitieron evaluar las causas de realización de ese proceso y hacer críticas de los defectos asociados a su funcionamiento o aplicación, puesto que las entrevistas semiestructuradas suministran un instrumento válido para combinar la fiabilidad científica con la posible carga subjetiva de las descripciones cualitativas. Se entrevistaron 13 personas del DMQ, cubriendo los principales niveles jerárquicos y los sectores más importantes para una política territorial. La entrevista, siguiendo un enfoque multidisciplinar, cubrió una serie de preguntas abiertas sobre la gestión de la municipalidad, la organización política, la situación y actitudes del personal, las finanzas, el sistema de información, los materiales de trabajo y las infraestructuras, la eficiencia del proceso y de las actividades y las relaciones externas.

En algunos casos, se ha desarrollado más de una entrevista por persona para evaluar y contrastar las diferentes opiniones. De esa forma, se aseguraba la credibilidad de los entrevistados, puesto que se tenía en cuenta la transferencia de los conceptos, la dependencia de los actores locales y las posibles fuentes externas de confirmación de los resultados (Lincoln y Guba, 1985).

Paralelamente, se ha estado observando sobre el terreno el funcionamiento explícito de la municipalidad y sus repercusiones específicas en el día a día de la comunidad de Quito y alrededores, durante el año 2014.

La información recopilada fue catalogada y asignada a cada uno de los conceptos teóricos básicos establecidos. Posteriormente, se establecieron las relaciones entre los conceptos mediante un análisis cualitativo.

\section{Innovación social en el MDQM}

El proceso de establecimiento de innovaciones sociales en el DMQ ha seguido una estrategia top-down, basado en el desarrollo de un plan estratégico incluyendo aspectos tecnológicos, económicos y sociales (MDMQ, 2012b). Las acciones específicas de ese plan

\footnotetext{
“Visión de Futuro" Año 19, Volumen No 26 N 1, Enero - Junio 2022 - Pág 123 - 148

URL de la Revista: http://visiondefuturo.fce.unam.edu.ar/index.php/visiondefuturo/index

URL del Documento: https://visiondefuturo.fce.unam.edu.ar/index.php/visiondefuturo/issue/view/22

ISSN 1668 - 8708 - Versión en Línea

E-mail: revistacientifica@fce.unam.edu.ar
} 
son muestra de innovaciones sociales aunque las mayores innovaciones aparecen en el alto nivel de integración entre los componentes sectoriales y los diferentes proyectos parciales (MDMQ, 2012c) y proyectos particulares como la agenda digital (MDMQ, 2012a). El proceso de difusión se inicia en el equipo de gobierno (actor innovador) se trasmite por la municipalidad (actores principales de difusión) y luego se extiende a otras regiones (actores de adaptación) mostrando características innovadoras, puesto que sugiere un proceso inverso al establecido en los supuestos del modelo tradicional: se trata de innovaciones que se desarrollan en países en vías de desarrollo que tienen potencial para desplazarse hacia países desarrollados por presentar una concepción basada en su experiencia local tradicional que es desconocida en la ciencia geográfica desarrollada.

\section{Las universidades y el conocimiento local del DMQ}

Una parte del conocimiento local se manifiesta en su contacto con las distintas universidades del territorio. Las universidades teóricamente son una fuente de innovación (Lara et al., 2020). El Distrito Metropolitano concentra las mejores Universidades del país y, en particular, las únicas dos que tienen derecho al doctorado (FLACSO, Universidad Andina Simón Bolívar) y la Universidad pública más antigua del país (Universidad Central). También tiene una serie de universidades privadas reconocidas (Católica, San Francisco). Tradicionalmente, las instituciones académicas de Quito han sido consideradas de menor nivel que las de otros territorios cercanos, como es el caso de Lima o Bogotá. Como consecuencia de ello, el Alcalde apostó decididamente por el Instituto de la Ciudad como entidad de ciencia aplicada de apoyo a las políticas municipales (think tank), para desarrollar el plan y potenciar la excelencia de la estructura administrativa (Gierhake y Jardón, 2016). Por tanto, en la práctica,las universidades no fueron fuente de innovación en el caso del DMQ.

Las manifestaciones del conocimiento local ajenos a las universidades aparecen en la percepción compartida entre gobierno municipal y ciudadanía, y se refleja en los planes municipales de desarrollo participativos, en la interactuación de las administraciones locales con los ciudadanos y en el fomento de las actuaciones público-privadas. También se muestra en una cierta cultura medioambiental, puesto que Quito fue una de las primeras ciudades que introdujeron políticas ambientales en el Municipio (Alcaldía de Jamil Mahuad), que se han mantenido (Barrera, 2014).

\footnotetext{
"Visión de Futuro" Año 19, Volumen No 26 N 1, Enero - Junio 2022 - Pág 123 - 148 URL de la Revista: http://visiondefuturo.fce.unam.edu.ar/index.php/visiondefuturo/index

URL del Documento: https://visiondefuturo.fce.unam.edu.ar/index.php/visiondefuturo/issue/view/22

ISSN 1668 - 8708 - Versión en Línea

E-mail: revistacientifica@fce.unam.edu.ar
} 
Tabla 1: Indicadores de innovación social en DMQ

Indicadores relativos a factores de territorios creativos (ejemplos):

Infraestructura de Universidades/Centros de Investigación, Amplia plataforma social institucional para la presentación de expresiones culturales, Impacto magnético por ideas nuevas y conceptos generales, Intercambio cultural sobrepasando las capas de la sociedad. Demanda de valores públicos por parte de la Sociedad Civil. Apoyar espacios físicos que facilitan la interacción, Cultura de política local (Quito inteligente).

\section{Indicadores de Redes metropolitanas}

Elaborar mecanismos de gestión a nivel político administrativo. Construir redes comunales para afrontar retos de la globalización. Conseguir conocimiento nuevo a través de cooperación internacional, Estructurar conocimiento nuevo y evitar aislamiento de información, Aprovechar impacto multiplicador de zonas metropolitanas, Alta centralidad en la perspectiva institucional.

\section{Indicadores de Capital Intelectual / Conocimiento local (ejemplos):}

a) humano: formación de profesionales, capacidad de integración y liderazgo, coordinación, capacidad de emprender

b) estructural: mecanismos de monitoreo, actividades de incentivar diálogo, recuperar centralidad del ser humano, conocimiento tecnológico

c) relacional: amplias relaciones internacionales, comunicación / participación

Fuente: Elaboración propia

Trabajos previos han estudiado expresiones particulares de la innovación social y su relación con diferentes grupos de factores aparentemente claves para el nacimiento de este proceso de innovación social, un programa holístico de modernización de la administración del Municipio y la planificación e implementación de los diferentes componentes de este programa. En particular se han destacado la importancia del conocimiento local (Gierhake y Jardon, 2017); de los factores de territorios creativos (Gierhake y Fernández-Jardon, 2017) y de las redes metropolitanos (Jardon y Gierhake, 2018). Sin embargo, los diferentes efectos del conocimiento local especifico del territorio y del conocimiento local más genérico transmitido por las universidades no se analizó con detalle. Esa conexión es la que permitía pasar de la visión estática de existencia de conocimiento local hacia una visión dinámica de difusión de ese conocimiento y de la innovación asociada. Este es el aspecto en el que se centra este estudio. La tabla 1 recoge el resumen de los resultados de análisis previos en el caso del $\mathrm{DMQ}$, desde un enfoque estático. A lo largo de las siguientes líneas se analiza el proceso dinámico asociado a la difusión de la innovación, más específicamente.

Los elementos detectados en el análisis estático señalan aspectos que han ayudado a crear las innovaciones sociales y que posiblemente también actúen sobre el proceso de difusión de la innovación, por lo que van a sugerir posibles barreras a dicho proceso.

\footnotetext{
"Visión de Futuro" Año 19, Volumen No 26 No 1, Enero - Junio 2022 - Pág 123 - 148 URL de la Revista: http://visiondefuturo.fce.unam.edu.ar/index.php/visiondefuturo/index

URL del Documento: https://visiondefuturo.fce.unam.edu.ar/index.php/visiondefuturo/issue/view/22

ISSN 1668 - 8708 - Versión en Línea

E-mail: revistacientifica@fce.unam.edu.ar
} 
A partir de esos trabajos previos y con el objetivo de entender los avances y barreras en la primera etapa de la difusión de esta innovación, se expone un mapa esquematizado del DMQ (ver figura 1), mostrando la innovación social que se llevó a cabo. La figura presenta dos niveles de análisis tal como se comentó previamente. El primer nivel recoge un enfoque sectorial, más estático. En el centro, se encuentran los principales factores que determinan el nacimiento de la innovación: el capital intelectual y el territorio. La combinación de esos dos elementos da lugar a los territorios creativos y al conocimiento local de la población, elementos que también están en el nacimiento de la innovación. Complementariamente, aparecen las redes metropolitanas en las que participaba el DMQ, que han servido como base de conocimiento para la creación de la innovación y como elemento clave en la difusión internacional de la innovación. Alrededor del centro de la figura, se describen los principales actores, con la función que realizaron en la innovación, introduciendo el análisis dinámico con las relaciones establecidas para mantener y difundir la innovación en el territorio. Se resalta especialmente el papel del gobierno local, como actor principal, y de las universidades como principales instituciones complementarias para crear, sistematizar y difundir los conocimientos profesionales asociados a la innovación.

\footnotetext{
"Visión de Futuro" Año 19, Volumen N$^{0} 26$ N 1, Enero - Junio 2022 - Pág 123 - 148

URL de la Revista: http://visiondefuturo.fce.unam.edu.ar/index.php/visiondefuturo/index

URL del Documento: https://visiondefuturo.fce.unam.edu.ar/index.php/visiondefuturo/issue/view/22

ISSN 1668 - 8708 - Versión en Línea

E-mail: revistacientifica@fce.unam.edu.ar
} 


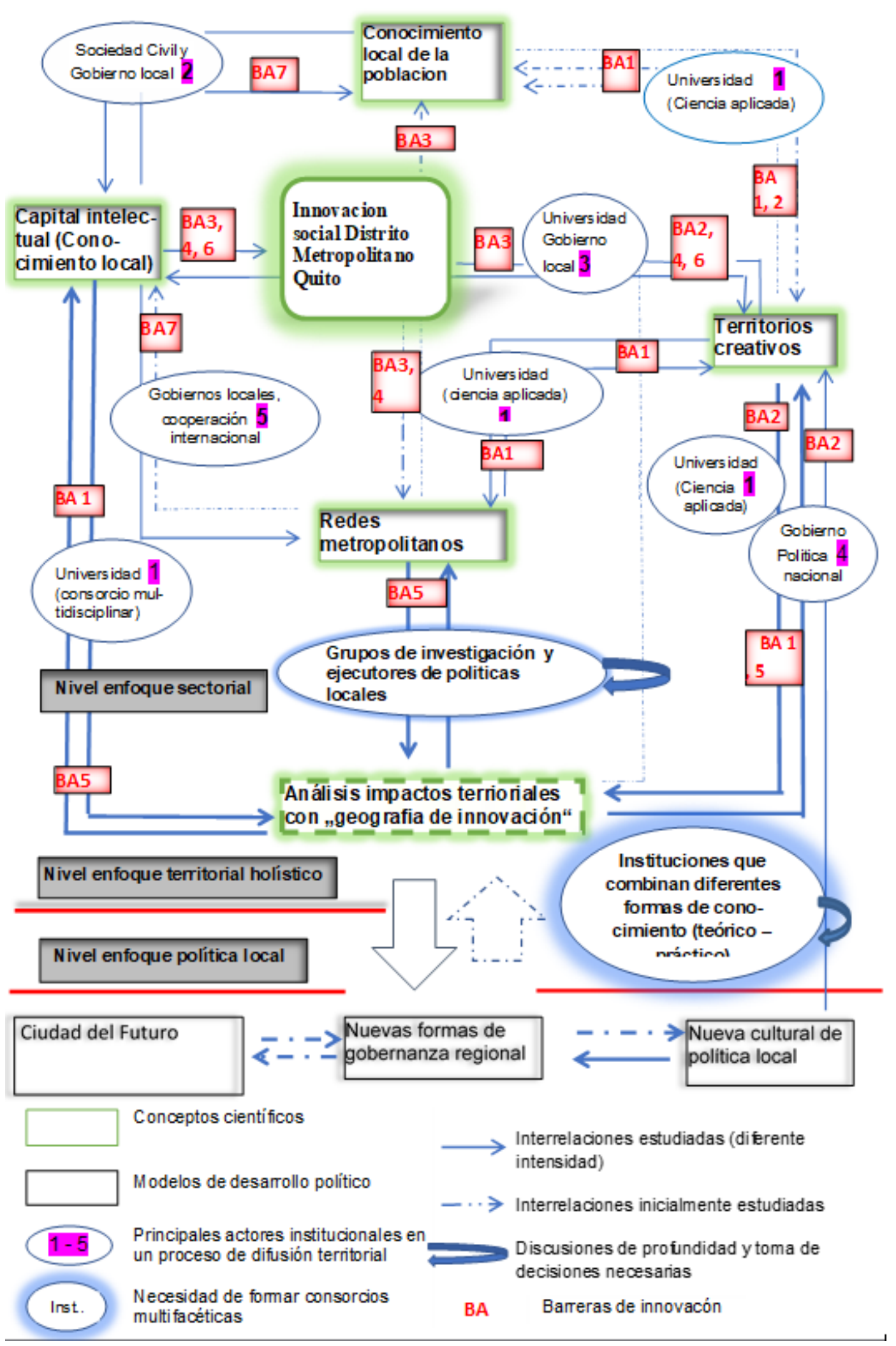

Figura 1: Barreras a la innovación social

Fuente: Elaboración propia

El nivel de enfoque territorial holístico del proceso de difusión de la innovación en el territorio introduce el análisis de los impactos territoriales con un enfoque de geografía de "Visión de Futuro" Año 19, Volumen No 26 No 1, Enero - Junio 2022 - Pág 123 - 148 URL de la Revista: http://visiondefuturo.fce.unam.edu.ar/index.php/visiondefuturo/index

URL del Documento: https://visiondefuturo.fce.unam.edu.ar/index.php/visiondefuturo/issue/view/22

ISSN 1668 - 8708 - Versión en Línea

E-mail: revistacientifica@fce.unam.edu.ar 
innovación. Esta visión dinámica permite introducir las barreras que se presentan en la interacción de los actores entre sí y con el territorio a la hora de difundir dicho proceso. La introducción de esas barreras sugiere posibles actuaciones políticas asociadas a la mejora y superación de dichas barreras.

Como consecuencia del análisis de la figura 1, se sugiere que los actores institucionales son esenciales en el proceso de la innovación social. De forma especial, se puede observar el papel que jugaron o deberían jugar las universidades locales en el territorio en ese proceso. En diferentes etapas del proceso, las universidades se combinaron con otros actores o instituciones formado figuras institucionales, las cuales se pueden agrupar por características compartidas, que se denominaron en el trabajo como aglomeración de actores. Cada una de estas aglomeraciones muestra ciertas características (que será denominada categoría) las cuales van a ser la base de las posibles barreras a la difusión de la innovación social por conocimientos no compartidos o no existentes. En particular, se destacan las siguientes:

Categoría 1: La coordinación entre la Universidad y la Sociedad civil, trabajando con ciencia aplicada: La ciencia aplicada como orientación científica es prácticamente desconocida en Ecuador, a pesar del hecho de que la Universidad en Ecuador tiene tres funciones: enseñanza, investigación y servicio a la sociedad. En particular, esta limitación de conocimientos se refiere también a la Geografía. De hecho, sólo existe una facultad en el país, y tiene una orientación clara hacia la geografía física. El tópico relativo al conocimiento local está interpretado como un aspecto rural, relativo a saberes ancestrales de los indígenas, sobre todo en temas de salud y medio ambiente. Consecuentemente, apenas existen investigaciones regionales, entendidas como investigaciones sobre el desarrollo sostenible del DMQ. Como respuesta a esa necesidad, se fortaleció el rol del Instituto de la Ciudad, pero en desconexión con las universidades locales.

Categoría 2: La Sociedad Civil en cooperación con Gobierno Local: EI MDMQ había logrado contratar varias personas de la sociedad civil, con experiencias profundas en otros sectores de la sociedad o con experiencia de trabajos en otros países. Esto sirvió para aprovechar el conocimiento local (de los profesionales trabajando en las jerarquías altas del MDMQ). Sin embargo, grupos de intereses económicos (por ejemplo, automovilistas, dueños de tiendas) no aceptaron el proceso de innovación, reclamando mantener privilegios anteriores (p. ej., estacionamiento libre de autos en espacio público o rechazar la construcción de bulevares con amplio espacio para peatones), limitando la colaboración con la sociedad civil.

\footnotetext{
"Visión de Futuro" Año 19, Volumen No 26 N 1, Enero - Junio 2022 - Pág 123 - 148 URL de la Revista: http://visiondefuturo.fce.unam.edu.ar/index.php/visiondefuturo/index

URL del Documento: https://visiondefuturo.fce.unam.edu.ar/index.php/visiondefuturo/issue/view/22

ISSN 1668 - 8708 - Versión en Línea

E-mail: revistacientifica@fce.unam.edu.ar
} 
Categoría 3: La relación entre las Universidades y el Gobierno Local: Hubo varios intentos de instalar plataformas y dialogo, pero no respondieron las universidades. La perspectiva territorial del MDMQ fue criticada en las universidades, sin conocer los contenidos del programa del MDMQ suficientemente, y tampoco sus posibles alcances internacionales en las políticas territoriales ya visibles.

Categoría 4: La interacción entre el Gobierno Local y el Gobierno Nacional: El hecho de que las autoridades de ambos gobiernos eran del mismo partido y compartían los mismos objetivos (Plan de Desarrollo, Ordenamiento Territorial), presentaba expectativas favorables. Además, existían ventajas debido a la cercanía geográfica de ambas sedes (por ejemplo, las oficinas de ambas instituciones se situaban a dos lados de la Plaza Principal). Este hecho facilitó la implementación de proyectos, pero no apoyó la nueva cultura de política local, una línea fundamental del MDMQ, puesto que la política del Gobierno Nacional era percibida como muy centralista por el MDMQ. En cuanto a las redes internacionales como mecanismo de difusión, el MDMQ estaba mejor posicionado, facilitando la difusión a nivel internacional, aspecto en el que el gobierno central no intervino.

Categoría 5: La conexión entre los gobiernos locales y la cooperación internacional: El MDMQ estaba en buena situación para priorizar la cooperación internacional, puesto que hubo un incremento de la oferta de cooperantes con interés en el municipio, (por ejemplo, la Cooperación alemana colaboró en la parte de planificación territorial). Además, hubo mucha cooperación a nivel de organismos municipales internacionales (Jardon y Gierhake, 2018). El MDMQ participó a en muchas redes, e incluso había asumido responsabilidades en algunas de ellas (vicepresidencia ...). Aparentemente, el DMQ estaba en una buena situación para facilitar el flujo de información (difusión de las ideas innovadoras, tanto para crear las innovaciones como para difundirlas internacionalmente) (Jardon y Gierhake, 2018), Por otro lado, la decisión de llevar a cabo la conferencia HABITAT 3 en Quito podría ser una muestra del posicionamiento institucional exitoso del municipio en el nivel internacional.

Como consecuencia de estas observaciones sobre actores, sus conocimientos y los respectivos manejos de conocimientos, se puede formular las siguientes barreras asociadas al papel de las universidades bien directamente, o bien indirectamente:

(BA1) Un primer aspecto que frenó la difusión de innovaciones fue la conexión entre la universidad y los diferentes actores de la innovación. Las universidades eran actores potenciales para promover la difusión de las ideas innovadoras hacia una parte de la sociedad, pero mantuvieron puntos de vista tradicionales que frenaron la difusión de la innovación. Por ejemplo, el programa nacional Prometeo, diseñado para modernizar las universidades e introducirlas en círculos de discusión internacional, posiblemente era una línea de difusión.

\footnotetext{
“Visión de Futuro" Año 19, Volumen No 26 N 1, Enero - Junio 2022 - Pág 123 - 148

URL de la Revista: http://visiondefuturo.fce.unam.edu.ar/index.php/visiondefuturo/index

URL del Documento: https://visiondefuturo.fce.unam.edu.ar/index.php/visiondefuturo/issue/view/22

ISSN 1668 - 8708 - Versión en Línea

E-mail: revistacientifica@fce.unam.edu.ar
} 
Sin embargo, no se pudieron crear impactos en la parte de políticas de innovación y territorio, entre otros aspectos, por la escasa conexión de las universidades con la sociedad y con el proceso de innovación.

(BA2) Un segundo aspecto se refiere al uso de diferentes conceptos o a la falta de conceptos relativos a la innovación social existentes en la universidad local, puesto que se conocen solamente territorios administrativos (estadísticas socio-administrativas) y territorios naturales (cuencas etc.). Los conceptos de territorios funcionales (definidos por ciertos factores que inducen otros procesos económicos o sociales) y de territorios virtuales (definidos por problemas potenciales compartidas por otras instituciones similares como, por ejemplo otros municipios, no necesariamente compartiendo los mismos límites) no eran conocidos por las instituciones universitarias locales. Por lo tanto, no se pudieron analizar adecuadamente aquellos movimientos territoriales que mostraron ciertas características socioeconómicas

(BA3) Las universidades locales presentan poca cultura de investigación multidisciplinar y de procesos territoriales relacionados con las barreras mencionadas anteriormente, y sobre todo, no existe apenas una orientación particular hacia los temas sociales-económicos. Con la administración Barrera se introdujo un programa de capacitación a través del Instituto de la Ciudad para fortalecer la ciencia aplicada orientada hacia necesidades del Municipio, pero no llegó a tener la altura científica que las universidades podrían haber desarrollado.

(BA4) La sociedad civil y el gobierno local no compartieron los conceptos políticos culturales: La administración Barrera introdujo varios conceptos nuevos relativos a la reorganización de la estructura administrativa, mejoras en flujos de información y nueva cultura de política local. Sin embargo, las jerarquías bajas de la administración no integraron adecuadamente esos conceptos, por lo que no se identificaron con este proceso, no lo apoyaron y como consecuencia, gran parte de las ideas no llegaron a una parte importante de lo población. Además, ciertos proyectos afectaron los intereses de grupos de interés económicos y políticos. Por ejemplo, los bulevares grandes para peatones en lugar de estacionamiento gratis para autos, o la red de ciclovías, o los locales permanentes con mejores condiciones sanitarias para ambulantes; o la implementación de multas por estacionar mal, etc.... Todo lo cual creó una ola de protestas, que frenaron la difusión de la innovación. La falta de una cultura cívica, a la que la universidad podría darle más apoyo, estaba en la raíz de esta limitación.

(BA5) La falta de monitoreo de impacto por parte del gobierno local. El desarrollo de la innovación en el DMQ solo presentaba un monitoreo de avance. Esto impedía seguir el desempeño de actividades complejas, actividades que incluyen percepciones nuevas y sobre

\footnotetext{
"Visión de Futuro" Año 19, Volumen No 26 N 1, Enero - Junio 2022 - Pág 123 - 148

URL de la Revista: http://visiondefuturo.fce.unam.edu.ar/index.php/visiondefuturo/index

URL del Documento: https://visiondefuturo.fce.unam.edu.ar/index.php/visiondefuturo/issue/view/22

ISSN 1668 - 8708 - Versión en Línea

E-mail: revistacientifica@fce.unam.edu.ar
} 
todo el cambio de percepciones, aspectos fundamentales en procesos de innovación social tan complejas como el caso del MDMQ. Este análisis requería una formación ampliamente multidisciplinar, poco conocida en Ecuador, posiblemente por los aspectos comentados previamente en relación con las universidades. Como consecuencia, no se disponía de información sobre los procesos. De hecho, en algunos aspectos avanzaron mejor de lo previsto, por ejemplo, los contactos municipales internacionales y la difusión de los conocimientos en estas redes, pero en otros, como la conexión con la ciudadanía, se quedaron cortos y no dio tiempo a reajustar el proceso. El actor principal del proceso de innovación (MDMQ) no tuvo tiempo para evaluar el alcance del proyecto, quizás porque no había pensado que era un proyecto innovador tan amplio. La falta de contactos adecuados con las universidades, que podría estar en condiciones de medir y comparar dichos avances limitó la comunicación adecuada sobre estos, y en un segundo paso, produjo limitaciones en el conocimiento sobre el avance en políticas innovadoras metropolitanas a nivel de la población local.

(BA6) El actor principal del proceso de la innovación (MDMQ) no tuvo tiempo para evaluar el alcance del proyecto en general, probablemente, porque no lo había pensado como un proyecto innovador amplio. Aunque identificó la falta de contactos adecuados con una academia en condiciones de medir y comparar los avances, y trató de paliarlo con el fortalecimiento del Instituto de la Ciudad, es esfuerzo no fue suficiente. Un limitado conocimiento en gran parte de la sociedad civil y unas costumbres reacias a los cambios en políticas metropolitanas innovadoras frenaron el proceso de difusión de la innovación. Los tiempos cortos de gobierno unido a la presión para implementar algo visible son problemas cotidianos en América Latina. En este escenario, un monitoreo de impacto representaría un instrumento importante, por ejemplo, de medir y comunicar avances. El hecho que este concepto de monitoreo de impacto no existía, refleja una barrera importante

(BA7) Como consecuencia de lo anterior, no se logró convencer de las ventajas amplias de la innovación social en el MDMQ a gran parte de la sociedad civil. Obviamente, el tiempo de desarrollo y aplicación del proyecto quedó corto, cuatro años, puesto que no hubo reelección del alcalde. El trabajo de relaciones públicas fue deficiente, habiendo muchos cambios en el departamento de relaciones públicas, se subestimaron los movimientos informales, con la creencia de que los buenos resultados iban a convencer. Como consecuencia, se pudo observar que los conocimientos sobre los avances eran limitados en la sociedad local, mezclados con idiosincrasias ambivalentes existentes en la cultura quiteña, donde se combina una cierta innovación cultural y social y una seriedad profesional con los aspectos informales de la cultura latinoamericana.

\footnotetext{
"Visión de Futuro" Año 19, Volumen No 26 N 1, Enero - Junio 2022 - Pág 123 - 148 URL de la Revista: http://visiondefuturo.fce.unam.edu.ar/index.php/visiondefuturo/index

URL del Documento: https://visiondefuturo.fce.unam.edu.ar/index.php/visiondefuturo/issue/view/22 


\section{CONCLUSIÓN}

El trabajo analiza el papel de las universidades en el proceso de la innovación social en el MDMQ desde un enfoque de conocimiento local, mostrando que el concepto original de los procesos geográficos de innovación y su conexión con el desarrollo territorial desde un enfoque de conocimiento local sirve como base para adaptarlo a entornos diferentes y analizar procesos complejos de innovación social. En combinación con el concepto de conocimiento local, el enfoque de geografía de innovación consigue un valor agregado, sugiriendo factores condicionantes del proceso de difusión de la innovación dentro de un entorno creativo.

La innovación social se manifiesta en el hecho de que la administración Augusto Barrera (MDMQ 2009-2014) haya implementado un proyecto holístico de modernización administrativa y de las estructuras territoriales del Distrito Metropolitano (esto incluye todos los aspectos del desarrollo sustentable). Este proyecto de modernización se refleja en el Plan Metropolitano de desarrollo - Quito 2021-2022, y una serie de documentos parciales / sectoriales, que se elaboraron en base de este Plan de Desarrollo y ha contado con todas las características de una innovación social; en el diseño de una agenda de trabajo para constituir una ciudad digital basada en un modelo colaborativo y en las características dinámicas de esta trasformación, mostrando elementos específicos de los procesos geográficos de innovación, tales como actores innovadores, actores de la difusión y actores de adaptación. Dicho proceso ha mostrado una originalidad, puesto que parte de países en vías de desarrollo y presenta un alto potencial para llegar a países desarrollados.

La estructura de los diferentes actores institucionales, su ubicación en relación con conceptos científicos que explican el avance de un proceso de innovación y las barreras identificadas permiten una serie de conclusiones:

En primer lugar, existen actores potenciales para promover la difusión de las ideas innovadoras a una parte de la sociedad. Entre ellos, merece mencionarse a las universidades, como un actor que reúne dos características importantes: por un lado, cierta sostenibilidad, independiente de cambios políticos; por otro lado, un catalizador potencial de novedades, porque concentran una población de educación superior, está más abierta a cambios que el promedio y teóricamente tiene contactos con el extranjero. Dentro del programa de desarrollo nacional ambicioso (Buen Vivir), se le adjudicó un rol importante a la modernización de las universidades (Programa Prometeo, Ciudad Universitaria Yachay), pero las universidades mantuvieron puntos de vista tradicionales. Posibles razones podrían ser la existencia de un cierto desfase temporal para implementar nuevos estructuras administrativas, una limitación de conceptos científicos y medidas para transformar esas estructuras en actividades "Visión de Futuro" Año 19, Volumen $\mathbf{N}^{\circ} \mathbf{2 6} \mathbf{N}^{0}$ 1, Enero - Junio 2022 - Pág 123 - 148
URL de la Revista: http://visiondefuturo.fce.unam.edu.ar/index.php/visiondefuturo/index
URL del Documento: https://visiondefuturo.fce.unam.edu.ar/index.php/visiondefuturo/issue/view/22
ISSN 1668 - 8708 - Versión en Línea
E-mail: revistacientifica@fce.unam.edu.ar 
universitarias; una percepción poco clara de una sobrecarga de actividades novedosas en la sociedad; y una percepción negativa sobre medidas decididas por el Gobierno Nacional que se expresó al nivel local en críticas acerca del Municipio.

Una innovación social ambiciosa como la que se llevó a cabo en el MDQM, dirigida a procesos que permitían aprovechar conocimientos, planificar, monitorear e introducir aspectos aprendidos en el proceso necesitaba una constelación de consorcio de actores, conformados especialmente por el gobierno local y la academia. La academia representa una figura institucional altamente diversa, entre universidades privadas y públicas, y entre diferentes facultades de una misma universidad. Esa diversidad era fundamental para crear y difundir la innovación. Hubiera sido esencial promover la innovación después de su introducción y primeras experiencias de difusión. Sin embargo, la organización de una estructura institucional favorable no pudo ser desarrollada hacia una estructura de funcionamiento, codirigiendo ese proceso de difusión. En las categorías de actor confluyen muchos aspectos diferentes que determinan su posición de aceptar o rechazar una innovación. Si un actor está mal informado (o rechaza novedades para mantener estructuras pasadas), se producen perdidas de fricción en la comunicación y en la forma de compartir los conocimientos necesarios. Dentro del concepto de innovación y de forma especial su difusión, esa falta de colaboración entre los actores fundamentales del proceso generó una barrera no visible (sin contar con los argumentos para pasar a un rechazo). Un ejemplo de este aspecto se muestra en el hecho de que el Municipio identificó los limitados conocimientos que tenía para analizar procesos territoriales, y puso empeño por superar esa imitación creando el Instituto de la Ciudad, pero dicha institución no fue suficiente para solucionar el problema de la difusión en el territorio.

En segundo lugar, existen aglomeraciones de actores con objetivos particulares y contrapuestos, en ciertas partes del proceso de difusión, señalando dos aspectos complementarios. Por una parte, no sorprenden las barreras presentes, puesto que se trata de un programa amplio de innovación social y es lógico que aparezcan frenos a ese proceso. Por otra parte, se muestra que los procesos asociados a la innovación social son diferentes a los procesos de innovación económica, aunque los actores actúan con racionalidades parecidas.

En tercer lugar, el trabajo muestra que en el DMQ existían conocimientos locales amplios y de alcance importante para iniciar un proceso de innovación social en el gobierno local y su jurisdicción. Basándose en los comentarios de todas las entrevistas, aparentemente el alcalde tenía la capacidad de reunir y estructurar estos conocimientos dentro de su administración. En la terminología de procesos territoriales de innovación, esta constelación de actores y situaciones permitió el la introducción y la difusión inicial de la innovación,

\footnotetext{
“Visión de Futuro" Año 19, Volumen No 26 N 1, Enero - Junio 2022 - Pág 123 - 148 URL de la Revista: http://visiondefuturo.fce.unam.edu.ar/index.php/visiondefuturo/index

URL del Documento: https://visiondefuturo.fce.unam.edu.ar/index.php/visiondefuturo/issue/view/22

ISSN 1668 - 8708 - Versión en Línea

E-mail: revistacientifica@fce.unam.edu.ar
} 
concentrándose en los niveles alto y medio del Municipio, y probablemente llegó a las mismas capas sociales de la sociedad civil y a las instituciones internacionales. En particular, llama la atención, que los avances en el nivel internacional, valorizados en otros contextos diferentes al de Ecuador, no se hayan podido traducir en impactos positivos para la difusión nacional de la innovación. Explicaciones posibles que requerirían investigaciones adicionales en los procesos de aceptación o rechazo de innovaciones sociales, sobre todo aquellas que tienen implicaciones políticas, y como se sobreponen a factores externos, tal como ha pasado en el caso de una creciente crítica hacia el Gobierno Nacional en Ecuador.

Un cuarto aspecto sugiere que, a nivel de una evaluación del proceso, desde una visión dinámica, se ha ampliado el modelo original de proceso de una innovación hacia una visión que incluye aspectos dinámicos (la difusión), resaltando posibles particularidades en el movimiento de la innovación social en un territorio, incluyendo sus actores y barreras potenciales. Este procesamiento encaja bien en la visión principal de ciencia aplicada, puesto que presenta unos resultados, los discute con usuarios potenciales, introduce nuevos conceptos y vuelve a analizar datos empíricos. Con respecto al nivel teórico, se ha mostrado como dos ciencias pueden contribuir a la interpretación de los procesos de difusión de una innovación. Se han identificado puntos de contacto entre las dos ciencias (economía y geografía social económica), llegando a un nivel de explicación más agregada.

Finalmente, se pudo mostrar, que existe una complementariedad entre conocimientos del área de Europa, con avances en teoría y construcción de modelos científicos, con ciertas ventajas en la acumulación de experiencias particulares, a nivel de ejecución de actividades en América Latina. De esa forma, se complementan las limitaciones que los conceptos teóricos presentan a nivel empírico, sobre todo mirando las regiones fuera de Europa y de América Latina, y las carencias existentes en algunos de esos territorios de un análisis más profundo sobre sus impactos positivos o limitaciones encontradas.

El trabajo presenta algunas limitaciones que deben considerarse a la hora de extender los resultados. Por una parte, el análisis esta realizado con indicadores cualitativos, si bien podrían representar las bases para desarrollar y comprobar esos resultados con indicadores cuantitativos más en adelante. Por otra parte, ha combinado el enfoque de dos ciencias, pero, para avanzar en el análisis, sería conveniente introducir otras ciencias con sus respectivos conceptos de investigación. En el grafico sugerimos algunos aspectos asociados a la ciencia política y relativos a la gobernanza local que se podrían tomar como punto de partida para precisar posibilidades de usar los resultados de innovación social y gobiernos locales a nivel de políticas locales, dejando abiertas nuevas líneas de investigación.

\footnotetext{
"Visión de Futuro" Año 19, Volumen No 26 N 1, Enero - Junio 2022 - Pág 123 - 148 URL de la Revista: http://visiondefuturo.fce.unam.edu.ar/index.php/visiondefuturo/index

URL del Documento: https://visiondefuturo.fce.unam.edu.ar/index.php/visiondefuturo/issue/view/22

ISSN 1668 - 8708 - Versión en Línea

E-mail: revistacientifica@fce.unam.edu.ar
} 


\section{REFERENCIAS}

Abbas, A., Avdic, A., Xiaobao, P., Hasan, M. M., y Ming, W. (2019). Universitygovernment collaboration for the generation and commercialization of new knowledge for use in industry. Journal of Innovation y Knowledge, 4(1), 23-31.

Barrera, A. (2014). Informe de Gestión del Alcalde de Quito, Augusto Barrera, 20092014. https://es.scribd.com/doc/221238761/Informe-de-Gestion-del-Alcalde-de-QuitoAugusto-Barrera-2009-2014

Battaglino, J. (2015). Políticos y militares en los gobiernos de la nueva izquierda sudamericana. Política y Gobierno, 22(1), 3-43.

Bernardino, S., y Santos, J. F. (2017). Local development through social and territorial innovation: An exploratory case study. CIRIEC-Espana Revista de Economia Publica, Social y Cooperativa, 1(90), 159-187.

Brökel, T. (2016). Wissens- und Innovationsgeographie in der Wirtschaftsförderung. Springer.

Brown, T., y Wyatt, J. (2010). Design Thinking for Social Innovation. Stanford Social Innovation Review, 8(1), 30-35.

Butzin, B. (2000). Netzwerke, Kreatives Milieu und Lernende Region. Zeitschrift Für Wirtschaftsgeographie, 44(3-4), 149-166.

Clark, J., Good, B., y Simmonds, P. (2008). Innovation in the public and third sectors. Innovation Index Working Paper: September.

Conejero Paz, E., y Redondo Lebrero, J. C. (2016). La innovación social desde el ámbito público: Social innovation in the public sphere: Concepts, experiences and obstacles. Gestión y Análisis de Políticas Públicas, 15(Enero-Junio), 22-40.

Dameri, R. P., y Ricciardi, F. (2015). Smart city intellectual capital: an emerging view of territorial systems innovation management. Journal of Intellectual Capital, 16(4), 860-887.

Denzin, N., y Lincoln, Y. (2000). Handbook of Qualitative Research. Sage Publications, 2000.

Eizaguirre Anglada, S. (2017). De la innovación social a la economía solidaria. Claves prácticas para el desarrollo de políticas públicas. CIRIEC-España, Revista de Economía Pública, Social y Cooperativa, 88, 201.

FEMP. (2011). Tercer Informe sobre las Políticas Locales de Lucha contra el Cambio Climático. Federación Española de Municipios y Provincias- Red Española de Ciudades Por El Clima. http://www.redciudadesclima.es/uploads/documentacion/85b83cd90c96f36d0042d9d1ac320 770.pdf

\footnotetext{
"Visión de Futuro" Año 19, Volumen No 26 N 1, Enero - Junio 2022 - Pág 123 - 148 URL de la Revista: http://visiondefuturo.fce.unam.edu.ar/index.php/visiondefuturo/index

URL del Documento: https://visiondefuturo.fce.unam.edu.ar/index.php/visiondefuturo/issue/view/22

ISSN 1668 - 8708 - Versión en Línea

E-mail: revistacientifica@fce.unam.edu.ar
} 
García-Flores, V., y Martos, L. P. (2019). Social innovation: Key factors for its development in the territories. CIRIEC-Espana Revista de Economia Publica, Social y Cooperativa, 97, 245-278.

Gierhake, K. (2015). Integrierter Umbau der Raumstruktur im Metropolitandistrikt Quito (Ecuador) - ein Ansatz zur Diskussion geographischer Innovationsforschung — ZEU (No. 67; Discussion Papers).

Gierhake, K., y Jardón, C. (2016). Territorium und Kreativität - Kommunale Entwicklungspolitik im Metropolitandistrikt Quito (2009 - 2014).

Gierhake, Klaus, y Fernández-Jardon, C. (2017). Indicadores de territorios creativos: una aplicación al Distrito Metropolitano Quito1. Visión de Futuro, 21(1), 146-155.

Gierhake, Klaus, y Jardon, C. M. (2017). Local knowledge and Creative environment: the case of the metropolitan district of Quito. Oikos Polis, Revista Latinoamericana de Ciencias Económicas y Sociales (RLCES), 2(1), 105-141.

Jardon, C.M., y Gierhake, K. (2017). Local knowledge as social innovation factor: The case of municipal district of Quito. Investigaciones Regionales, 2017(38).

Jardon, C.M., y Gierhake, K. (2018). Redes municipales como instrumento para difundir innovaciones sociales: el Distrito Metropolitano de Quito. Oikos Polis, Revista Latinoamericana, 3(2), 1-46.

Jardon, Carlos M, y Gierhake, K. (2020). Innovación social y territorio en municipios: el caso del Distrito Metropolitano de Quito Social innovation and territory in municipalities: The Distrito Metropolitano de Quito case. Perfiles Latinoamericanos, 28(55), 301-324.

Ketelhöhn, N., y Ogliastri, E. (2013). Introduction: innovation in Latin America. Academia Revista Latinoamericana de Administración, 26(1), 12-32.

Koch, P., y Hauknes, J. (2005). On innovation in the public sector--today and beyond.

Lara, G., Llach, J., y Arbussa, A. (2020). Innovation performance of the firms that have cooperated with universities and research institutes in Spain. International Journal of Innovation Management, 24(06), 2050053.

Lau, A. K. W., y Lo, W. (2015). Regional innovation system, absorptive capacity and innovation performance: An empirical study. Technological Forecasting and Social Change, 92, 99-114.

Li, N., y Toppinen, A. (2011). Corporate responsibility and sustainable competitive advantage in forest-based industry: Complementary or conflicting goals? Forest Policy and Economics, 13(2), 113-123.

Lincoln, Y., y Guba, E. (1985). Naturalistic inquiry. Sage.

\footnotetext{
"Visión de Futuro" Año 19, Volumen No 26 N 1, Enero - Junio 2022 - Pág 123 - 148

URL de la Revista: http://visiondefuturo.fce.unam.edu.ar/index.php/visiondefuturo/index

URL del Documento: https://visiondefuturo.fce.unam.edu.ar/index.php/visiondefuturo/issue/view/22

ISSN 1668 - 8708 - Versión en Línea

E-mail: revistacientifica@fce.unam.edu.ar
} 
Manosalvas, M. (2014). Buen vivir o sumak kawsay. En busca de nuevos referenciales para la acción pública en Ecuador. Iconos. Revista de Ciencias Sociales, 49(1), 101-121.

MDMQ. (2012a).Agenda_Digital_Quito_2022. Municipio de Quito Distrito Metropolitano. http://www.quitodigital.gob.ec/wpcontent/uploads/2013/10/Agenda_Digital_Quito_2022_Resumen.pdf

MDMQ. (2012b). ORDM-0263: Régimen administrativo de fomento a las innovaciones tecnológicas y creaciones originales en el distrito metropolitano de Quito. Municipalidad Del Distrito Metropolitano de Quito. http://www7.quito.gob.ec/mdmq_ordenanzas/Ordenanzas/ORDENANZAS MUNICIPALES 2012/ORDM-0263 RÉGIMEN ADMINISTRATIVO DE FOMENTO A LAS INNOVACIONES TECNOLÓGICAS Y CREACIONES ORIGINALES EN EL DISTRITO METROPOLITANO DE QUITO.pdf

MDMQ. (2012c). Plan metropolitano de desarrollo 2012 - 2022. Municipalidad Del Distrito Metropolitano de Quito. http://www.centroculturalquito.com/imagesFTP/13644.Plan_de_Desarrollo_Local_2012_2022.pdf

Mendes, A., Batista, A., Fernandes, L., Macedo, P., Pinto, F., Rebelo, L., Ribeiro, M., Ribeiro, R., Sottomayor, M., Tavares, M., y Verdelho, V. (2012). Barriers to Social Innovation. Brussels: European Commission, DG Research, April 2016.

Mingxing, L., Asunka, B. A., Jialu, S., Cheng, H., Ming, W., y Yuxiao, W. (2020). Sustaining Corporate Innovation Through University-Industry Collaborative Research: Evidence from the Jiangsu University of China. Journal of Industrial Integration and Management, 05(02), 235-252.

Morales Gutiérrez, A. C. (2009). Innovación social: un ámbito de interés para los servicios sociales. EKAINA, 45(Junio), 151-178.

OECD. (2005). The Measurement of Scientific and Technological Activities: Proposed Guidelines for Collecting and Interpreting Technological Innovation Data. OSLO Manual.

Oeij, P. R. A., van der Torre, W., Vaas, F., y Dhondt, S. (2019). Understanding social innovation as an innovation process: Applying the innovation journey model. Journal of Business Research, 101, 243-254.

Oganisjana, K., Surikova, S., y Laizāns, T. (2015). Factors influencing social innovation processes in Latvia: qualitative research perspective. Entrepreneurship and Sustainability Issues, 3(2), 186-197.

Ogden, A. E., y Innes, J. L. (2009). Adapting to climate change in the southwest Yukon: Locally identified research and monitoring needs to support decision making on sustainable forest management. 62(2), 159-174. 
Placet, M., Anderson, R., y Fowler, K. M. (2005). Strategies for Sustainability. ResearchTechnology Management, 48(1), 32-41.

Rodríguez Herrera, A., y Alvarado Ugarte, H. (2008). Claves de la innovación social en América Latina y el Caribe. CEPAL.

Sáez, M. A. (2017). Innovación social y capacidad institucional en Latinoamérica. Revista Del CLAD Reforma y Democracia, 67(1), 33-68.

Seleim, A., y Bontis, N. (2013). National Intellectual Capital and Economic Performance: Empirical Evidence from Developing Countries. Knowledge and Process Management, 20(3), $131-140$.

UN. (1992). División de Desarrollo Sostenible de las Naciones Unidas. Division de Desarrollo Sostenible.

http://www.un.org/spanish/esa/sustdev/agenda21/agenda21spchapter2.htm

Wethinq. (2016). 41 Inspiring Examples of Social Innovation. https://www.wethinq.com/en/blog/2014/02/18/32-Inspiring-Examples-of-SocialInnovation.html

Williamson, T., Hesseln, H., y Johnston, M. (2012). Adaptive capacity deficits and adaptive capacity of economic systems in climate change vulnerability assessment. Forest Policy and Economics, 15(null), 160-166.

Wolfe, S. E. (2009). A social innovation framework for water demand management policy: Practitioners' capabilities, capacity, collaboration, and commitment. In Society and Natural Resources (Vol. 22, Issue 5, pp. 474-483).

\section{RESUMEN BIBLIOGRÁFICO}

\section{Klaus Gierhake}

Dr. rer. nat, Universidad Marburg / Alemania. Fundador empresa CRELA, consultor independiente de varios organismos internacionales. Intereses de investigación: Innovación social en gobiernos locales, factores de territorios creativos (aparte de lo económico) conocimiento complementario entre Europa y América Latina en temas de territorio, innovación social; análisis institucional gobierno local y sociedad civil, monitoreo de impacto políticas públicas.

\section{Carlos María Fernandez Jardon}

Doctor en Economía por la Universidad de Navarra y doctor en matemáticas por la Universidad de Navarra. MBA executive de Caixanova Business School, Master en gestión del conocimiento por la Universidad Complutense de Madrid. Es Catedrático de Econometría en la Facultad de Ciencias Económicas y Empresariales de la Universidad de Vigo. Ha realizado numerosas investigaciones en el campo de la Economía aplicada y de investigación de mercados. Colaborador en diferentes proyectos de investigación en empresas gallegas y en proyectos de cooperación en Rusia, en Argentina y Paraguay, para el desarrollo de planes estratégicos en cadenas empresariales y proceso de integración

\footnotetext{
"Visión de Futuro" Año 19, Volumen No 26 N 1, Enero - Junio 2022 - Pág 123 - 148

URL de la Revista: http://visiondefuturo.fce.unam.edu.ar/index.php/visiondefuturo/index

URL del Documento: https://visiondefuturo.fce.unam.edu.ar/index.php/visiondefuturo/issue/view/22

ISSN 1668 - 8708 - Versión en Línea

E-mail: revistacientifica@fce.unam.edu.ar
} 
productiva transfronteriza. Tiene diferentes publicaciones en temas especializados capital intelectual, innovación y pequeñas empresas en revistas internacionales como el Journal of Intellectual capital, International Journal of Emerging markets, Business Review Quaterly, entre otras. 